\title{
Exact solutions of coupled burgers equation with time-and space-fractional derivative
}

\author{
Salih M. Elzaki \\ Math.Dept. Sudan University of Science and Technology on secondment to Shaqra University Kingdom of Saudi Arabia \\ E-mail: Salih.alzaki@gmail.com
}

Copyright $\odot 2015$ Salih M. Elzaki. This is an open access article distributed under the Creative Commons Attribution License, which permits unrestricted use, distribution, and reproduction in any medium, provided the original work is properly cited.

\begin{abstract}
In this paper, He's projected differential transform method (PDTM) has been used to obtain solution nonlinear coupled Burgers equation. This method involves less computational work and can, thus, be easily applied to initial value problems. (PDTM) is used to determine the exact solutions of some nonlinear time and space--fractional partial differential equations. A number of illustrative examples are provided and compared with the other methods. The numerical results obtained by these examples are found to be the same.
\end{abstract}

Keywords: Coupled Burgers Equation with Time and Space-Fractional Derivative the Projected Differential Transforms Method; Numerical Method.

\section{Introduction}

Projected differential transform method is numerical solution technique that is based on Taylor series expansion which constructs an analytical solution in the form of a polynomial .However; projected differential transform method obtained a polynomial series solution by means of an iterative procedure. [3], [10], [12].

There are several approaches to the generalization of the notion of differentiation to fractional orders e.g Riemann Liouville. Grunwald - Letnikow. Caputo and generalized functions approach. Riemann-Lioville fractional derivative is mostly employed by mathematicians but this approach is not suitable for real world physical problems since it requires the definition of fractional order initial conditions, which has the advantage of defining integer order initial conditions which have no physically meaning full explanation as yet. Caputo introduced an alternative definition, for fractional order differential equations. Unlike the Riemann Liouville approach which derives its definition from repeated integration, the Grunwald Letnikow formulation approaches the problem from the derivative perspective. This approach is mostly used in numerical algorithms.

Moreover, there are several techniques for the solution of fractional differential equations. The most commonly used ones are Adomian decomposition method (ADM), variational iteration method (VIM), Fractional difference method (FDM) and power series method. Also there are some classical solution techniques. e.g Laplace transform method, fractional Green's function method, Mellin transform method and method of orthogonal polynomials. Among these solution techniques, the power series method is the most transparent method of solution of fractional differential and integral equations.

There are several definitions of a fractional derivative of order $\alpha>0$. The two most commonly used definitions are the ones by Riemann - Liouville and Capout. Each definition uses Riemann-Liouville fractional integration and derivations of whole order. The difference between the two definitions lies in the order of evaluation Riemann-Louville fractional integral of order $\alpha$ is defined as [1], [4], [5], [6], [8], [13].

$J_{x_{0}}^{\alpha} f(X, x)=\frac{1}{\Gamma(\alpha)} \int_{x_{0}}^{x}(x-t)^{\alpha-1} f(X, t) d t, \quad \alpha>0, x>0, X=X\left(x_{1}, x_{2}, \ldots, x_{n}\right)$

The next two equations define Riemann Liouville and caputo fractional derivatives will of order $\alpha$, respectively 


$$
\begin{aligned}
& D_{x_{o}}^{\alpha} f(X, x)=\frac{\partial^{m}}{\partial x^{m}}\left[J^{m-\alpha} f(X, x)\right] \\
& D_{* x_{o}}^{\alpha} f(X, x)=J^{m-\alpha}\left[\frac{\partial^{m}}{\partial x^{m}} f(X, x)\right]
\end{aligned}
$$

Where $m-1<\alpha>m$ and $\mathrm{m} \in \mathrm{N}$.For now, the Caputo fractional derivative will be denoted by $D_{* x_{0}}^{x}$ to maintain a clear distinction with the Riemann - Liouville fractional derivative. The Caputo fractional derivative first computes an ordinary derivative followed by a fractional integral to achieve the desired order of fractional derivative. The Riemann Liouville fractional derivative is computed in the reverse order we have chosen to use the Coputo fractional derivative because it allows traditional initial and boundary conditions to include in the formulation of the problem, but for homogeneous initial condition assumption, these two operators coincide [2], [7], [9], [11].

\section{Fractional projected differential transform method}

We introduce the fractional projected differential transform method used in the sequent to obtain approximate analytical solutions for a fractional oscillator this method has been developed by Arikoglu and Ozkol as follows.

The fractional differentiation in Riemann - liouville sense is defined by

$$
D_{x_{0}}^{q} f(X, x)=\frac{1}{\Gamma(m-q)} \frac{\partial^{m}}{\partial x^{m}}\left[\int_{x_{0}}^{x} \frac{f(X, t)}{(x-t)^{1+q-m}} d t\right]
$$

For $\mathrm{m}-1 \leq \mathrm{q} \leq \mathrm{m}, \mathrm{m} \in \mathrm{N}$, and $\mathrm{x}>\mathrm{x}_{0}$ let us expand the analytical and continuous function $f(X, x)$ in terms of a fractional power series as follows:

$f(X, x)=\sum_{k=O}^{\infty} f(X, k \alpha q)\left(x-x_{o}\right)^{\alpha k}$

Where $\alpha$ is the order of fractional and $f(X, k)$ is the fractional projected differential transform of $f(X, x)$.

In order to avoid fractional initial and boundary conditions, we define the fractional derivative in the caputo sense. The relation between the Riemann- Liovuille operator and Caputo operator is given by

$$
D_{{ }^{*} x_{o}}^{q} f(X, x)=D_{x_{o}}^{q}\left[f(X, x)-\sum_{k=o}^{m-1} \frac{f(k)\left(X, x_{O}\right)}{k !}\left(x-x_{o}\right)^{k}\right]
$$

Setting in eq (4) and using eq (6) we obtain fractional derivative the caputo sense as follows

$$
D_{* x_{O}}^{q} f(X, x)=\frac{1}{\Gamma(m-q)} \frac{\partial^{m}}{\partial x^{m}} \int_{x_{O}}^{x}\left[\frac{f(X, t)-\sum_{k=O}^{m-1} \frac{f(k)\left(X, x_{O}\right)}{k i}\left(t-x_{O}\right)^{k}}{(x-t)^{1+q-m}}\right] d t
$$

Since the initial conditions are implemented to the integer order derivatives, the transformations of the initial conditions are defined as follows:

$$
F(X, k)=\left\{\begin{array}{cc}
\frac{1}{(k / \alpha) !}\left[\frac{\frac{k}{\alpha} f(X, x)}{\partial x \frac{k}{\alpha}}\right]_{x=x_{o}} \text { if } \frac{k}{\alpha} \in z^{+} \text {for } k=0,1, \ldots . q(q \alpha-1) \\
0 & \text { if } \quad \frac{k}{\alpha} \notin z^{+}
\end{array}\right.
$$

Where $\mathrm{q}$ is the order of fractional differential equation considered

Theorems:

(1) If $f(X, x)=g(X, x) \pm h(X, x)$ Then $f(X, k)=g(X, k) \pm h(X, k)$

(2) If $f(X, x)=c g(X, x)$ Then $f(X, k)=c g(X, k)$, c is constant

(3) If $f(X, x)=g(X, x) h(X, x)$ Then $F(X, k)=\sum_{l=0}^{k} G(X, l) H(X, k-l)$ 
(4)If $f(X, x)=g_{1}(X, x) g_{2}(X, x) \ldots \ldots \ldots \ldots g_{n}(X, x)$ Then

$f(X, k)=\sum_{k_{n-1}=0}^{k} \sum_{k_{n-2}=0}^{k_{n-1}} \ldots \sum_{k_{2}=0}^{k_{3}} \sum_{k_{1}=0}^{k_{2}} G_{1}\left(X, k_{1}\right) G_{2}\left(X, k_{2}-k_{1}\right) G_{n-1}\left(X, k_{n-1}-k_{n-2}\right) G_{n}\left(X, k-k_{n-1}\right)$

(5) If $f(X, x)=D_{x_{o}}^{q}[g(X, x)]$ Then $F(X, k)=\frac{\Gamma(q+1+k / \alpha)}{\Gamma(1+k / \alpha)} G(X, k+\alpha q)$

(6) If $f(x, t)=u D_{t}^{\alpha} v$ Then $f(x, k)=\sum_{m=0}^{k} \frac{\Gamma\left(1+\alpha+\frac{k}{q}\right)}{\Gamma\left(1+\frac{k}{q}\right)} u(x, m) v(x, k+\alpha q-m)$

(7) If $f(X, x)=\left[\frac{\partial^{q_{1}}}{\partial x^{q_{1}}} g_{1}(X, x)\right]\left[\frac{\partial^{q_{2}}}{\partial x^{q_{2}}} g_{2}(X, x)\right] \ldots \ldots . .\left[\frac{\partial^{q_{n}}}{\partial x^{q_{n}}} g_{n}(X, x)\right]$ Then

$F(X, k)=\sum_{k_{n-1}=o}^{k} \sum_{k_{n-2}=o}^{k_{n-1}} \ldots . . \sum_{k_{2}=o}^{k_{3}} \sum_{k_{1}=o}^{k_{2}} \frac{\Gamma\left(q_{1}+1+k_{1} / \alpha\right)}{\Gamma(1+k / \alpha)} \frac{\Gamma\left(q_{2}+1+\left(k_{2}-k_{1}\right) / \alpha\right)}{\Gamma\left(1+\left(k_{2}-k_{1}\right) / \alpha\right.} \ldots \ldots \ldots$

$\times \frac{\Gamma\left(q_{n}+\left(k_{1}+k_{n-1}\right) / \alpha\right)}{\Gamma\left(1+\left(k-k_{n-1}\right) / \alpha\right)} G_{1}\left(X, k_{1}+\alpha q_{1}\right) G_{2}\left(X, k_{2}-k_{1}+\alpha q_{2}\right) \ldots . . G_{n}\left(X, k-k_{n-1}+\alpha q_{n}\right)$

Where

$\alpha, q_{i} \in z^{+}$for $i=0,1,2, \ldots \ldots, n$

(8)If $f(X, x)=\left(x-x_{0}\right)^{p}$ Then $f(X, k)=\delta(k-\alpha p)$

Where $\delta(X, k)=\left\{\begin{array}{lll}1 & \text { if } & k=0 \\ 0 & \text { if } & k \neq 0\end{array}\right.$

\section{Analysis of the method:}

Consider the Coupled Burgers equation with time-and space-fractional derivatives

$\left\{\begin{array}{l}D_{t}^{\alpha} u=D_{x}^{2 \alpha} u+2 u D_{x}^{\alpha} u-D_{x}^{\alpha}(u v) \\ D_{t}^{\alpha} v=D_{x}^{2 \alpha} v+2 v D_{x}^{\alpha} v-D_{x}^{\alpha}(u v)\end{array} \quad, 0<\alpha \leq 1\right.$

With the initial condition $u(x, 0)=f(x), v(x, 0)=g(x)$

Taking the Projected differential transform method of equation (9) we obtain

$$
\left\{\begin{aligned}
\frac{\Gamma\left(1+\alpha+\frac{k}{q}\right)}{\Gamma\left(1+\frac{k}{q}\right)} u(x, k+\alpha q) & =D_{x}^{2 \alpha} u(x, k)+2 \sum_{m=0}^{k} u(x, m) D_{x}^{\alpha} u(x, k-m) \\
& -D_{x}^{\alpha}\left(\sum_{m=0}^{k} u(x, m) v(x, k-m)\right) \\
\frac{\Gamma\left(1+\alpha+\frac{k}{q}\right)}{\Gamma\left(1+\frac{k}{q}\right)} v(x, k+\alpha q) & =D_{x}^{2 \alpha} v(x, k)+2 \sum_{m=0}^{k} v(x, m) D_{x}^{\alpha} v(x, k-m) \\
- & D_{x}^{\alpha}\left(\sum_{m=0}^{k} u(x, m) v(x, k-m)\right) \quad, k=0, \alpha q, 2 \alpha q, \ldots . .
\end{aligned}\right.
$$




$$
\left\{\begin{array}{r}
u(x, k \alpha q)=\frac{\Gamma(1+(k-1) \alpha)}{\Gamma(1+\alpha k)}\left[D_{x}^{2 \alpha} u(x,(k-1) \alpha q)+2 \sum_{m=0}^{(k-1) \alpha q} u(x, m) D_{x}^{\alpha} u(x,(k-1) \alpha q-m)\right. \\
\left.-D_{x}^{\alpha}\left(\sum_{m=0}^{(k-1) \alpha q} u(x, m) v(x,(k-1) \alpha q-m)\right)\right] \\
v(x, k \alpha q)=\frac{\Gamma(1+(k-1) \alpha)}{\Gamma(1+\alpha k)}\left[\begin{array}{c}
D_{x}^{2 \alpha} v(x,(k-1) \alpha q)+2 \sum_{m=0}^{(k-1) \alpha q} v(x, m) D_{x}^{\alpha} v(x,(k-1) \alpha q-m) \\
\left.-D_{x}^{\alpha}\left(\sum_{m=0}^{(k-1) \alpha q} u(x, m) v(x,(k-1) \alpha q-m)\right)\right]
\end{array}\right.
\end{array}\right.
$$

Substituting $u(X, k \alpha q), v(X, k \alpha q)$ into equation (5) we get

$$
\begin{aligned}
& u(x, t)=f(x)+\sum_{k=1}^{\infty} \frac{1}{k !} \frac{\Gamma(1+(k-1) \alpha)}{\Gamma(1+\alpha k)}\left[D_{x}^{2 \alpha} u(x,(k-1) \alpha q)+\right. \\
& \left.2 \sum_{m=0}^{(k-1) \alpha q} u(x, m) D_{x}^{\alpha} u(x,(k-1) \alpha q-m)-D_{x}^{\alpha}\left(\sum_{m=0}^{(k-1) \alpha q} u(x, m) v(x,(k-1) \alpha q-m)\right)\right] t \alpha k \\
& v(x, t)=g(x)+\sum_{k=1}^{\infty} \frac{1}{k !} \frac{\Gamma(1+(k-1) \alpha)}{\Gamma(1+\alpha k)}\left[D_{x}^{2 \alpha} v(x,(k-1) \alpha q)+\right. \\
& \left.\quad 2 \sum_{m=0}^{(k-1) \alpha q} v(x, m) D_{x}^{\alpha} v(x,(k-1) \alpha q-m)-D_{x}^{\alpha}\left(\sum_{m=0}^{(k-1) \alpha q} u(x, m) v(x,(k-1) \alpha q-m)\right)\right] t
\end{aligned}
$$

\section{Application}

\section{Coupled Burgers equation:}

1) Coupled Burgers equation with time-fractional derivative:

\section{Example:}

$$
\left\{\begin{array}{l}
D_{t}^{\alpha} u=u_{x x}+2 u u_{x}-(u v)_{x} \quad, 0<\alpha \leq 1 \\
D_{t}^{\alpha} v=v_{x x}+2 v v_{x}-(u v)_{x}
\end{array}\right.
$$

With the initial condition $u(x, 0)=\sin x, v(x, 0)=\sin x$

Taking the Projected differential transform method of equation (10) we obtain

$$
\begin{aligned}
& \left(\frac{\Gamma\left(1+\alpha+\frac{k}{q}\right)}{\Gamma\left(1+\frac{k}{q}\right)} u(x, k+\alpha q)=u_{x x}(x, k)+2 \sum_{m=0}^{k} u(x, m) u_{x}(x, k-m)-\left(\sum_{m=0}^{k} u(x, m) v(x, k-m)\right)_{x}\right. \\
& \frac{\Gamma\left(1+\alpha+\frac{k}{q}\right)}{\Gamma\left(1+\frac{k}{q}\right)} v(x, k+\alpha q)=v_{x x}(x, k)+2 \sum_{m=0}^{k} v(x, m) v_{x}(x, k-m)-\left(\sum_{m=0}^{k} u(x, m) v(x, k-m)\right)_{x} \\
& k=0, \alpha q, 2 \alpha q, \ldots . \\
& u(x, k \alpha q)=\frac{\Gamma(1+(k-1) \alpha)}{\Gamma(1+\alpha k)}\left[u_{x x}(x,(k-1) \alpha q)+2 \sum_{m=0}^{(k-1) \alpha q} u(x, m) u_{x}(x,(k-1) \alpha q-m)\right. \\
& \left.-\left(\sum_{m=0}^{(k-1) \alpha q} u(x, m) v(x,(k-1) \alpha q-m)\right)_{x}\right] \\
& v(x, k \alpha q)=\frac{\Gamma(1+(k-1) \alpha)}{\Gamma(1+\alpha k)}\left[v_{x x}(x,(k-1) \alpha q)+2 \sum_{m=0}^{(k-1) \alpha q} v(x, m) v_{x}(x,(k-1) \alpha q-m)\right. \\
& \left.-\left(\sum_{m=0}^{(k-1) \alpha q} u(x, m) v(x,(k-1) \alpha q-m)\right)_{x}\right]
\end{aligned}
$$




$$
\begin{aligned}
& u(x, \alpha q)=\frac{1}{\Gamma(1+\alpha)}\left[u_{x x}(x, 0)+2 u(x, 0) u_{x}(x, 0)-(u(x, 0) v(x, 0))_{x}\right]=\frac{-\sin x}{\Gamma(1+\alpha)} \\
& v(x, \alpha q)=\frac{1}{\Gamma(1+\alpha)}\left[v_{x x}(x, 0)+2 v(x, 0) v_{x}(x, 0)-(u(x, 0) v(x, 0))_{x}\right]=\frac{-\sin x}{\Gamma(1+\alpha)} \\
& u(x, 2 \alpha q)=\frac{\Gamma(1+\alpha)}{\Gamma(1+2 \alpha)}\left[u_{x x}(x, \alpha q)+2 u(x, 0) u_{x}(x, \alpha q)+2 u(x, \alpha q) u_{x}(x, 0)\right. \\
& \left.-(u(x, 0) v(x, \alpha q)+u(x, \alpha q) v(x, 0))_{x}\right]=\frac{\sin x}{\Gamma(1+2 \alpha)} \\
& v(x, 2 \alpha q)=\frac{\Gamma(1+\alpha)}{\Gamma(1+2 \alpha)}\left[v_{x x}(x, \alpha q)+2 v(x, 0) v_{x}(x, \alpha q)+2 v(x, \alpha q) v_{x}(x, 0)\right. \\
& \left.-(u(x, 0) v(x, \alpha q)+u(x, \alpha q) v(x, 0))_{x}\right]=\frac{\sin x}{\Gamma(1+2 \alpha)} \\
& u(x, 3 \alpha q)=\frac{\Gamma(1+2 \alpha)}{\Gamma(1+3 \alpha)}\left[u_{x x}(x, 2 \alpha q)+2 u(x, 0) u_{x}(x, 2 \alpha q)+2 u(x, \alpha q) u_{x}(x, \alpha q)+\right. \\
& \left.2 u(x, 2 \alpha q) u_{x}(x, 0)-(u(x, 0) v(x, 2 \alpha q)+u(x, \alpha q) v(x, \alpha q)+u(x, 2 \alpha q) v(x, 0))_{x}\right]=\frac{-\sin x}{\Gamma(1+3 \alpha)} \\
& u(x, 3 \alpha q)=\frac{\Gamma(1+2 \alpha)}{\Gamma(1+3 \alpha)}\left[u_{x x}(x, 2 \alpha q)+2 u(x, 0) u_{x}(x, 2 \alpha q)+2 u(x, \alpha q) u_{x}(x, \alpha q)+\right. \\
& \left.2 u(x, 2 \alpha q) u_{x}(x, 0)-(u(x, 0) v(x, 2 \alpha q)+u(x, \alpha q) v(x, \alpha q)+u(x, 2 \alpha q) v(x, 0))_{x}\right]=\frac{-\sin x}{\Gamma(1+3 \alpha)}
\end{aligned}
$$

And soon in general $u(x, k \alpha q)=\frac{(-1)^{k} \sin x}{\Gamma(1+k \alpha)}, v(x, k \alpha q)=\frac{(-1)^{k} \sin x}{\Gamma(1+k \alpha)}$

Substituting $u(X, k \alpha q), v(X, k \alpha q)$ into equation (5) we get

$$
\begin{aligned}
& u(x, t)=\sum_{k=0}^{\infty} \frac{(-1)^{k} \sin x}{\Gamma(1+k \alpha)} t^{\alpha k}=(\sin x) \sum_{k=0}^{\infty} \frac{(-t \alpha)^{k}}{(k \alpha) !}=(\sin x)\left(E_{\alpha}(-t)\right) \\
& v(x, t)=\sum_{k=0}^{\infty} \frac{(-1)^{k} \sin x}{\Gamma(1+k \alpha)} t^{\alpha k}=(\sin x) \sum_{k=0}^{\infty} \frac{(-t \alpha)^{k}}{(k \alpha) !}=(\sin x)\left(E_{\alpha}(-t)\right) \\
& \alpha \rightarrow 1 \quad u(X, t)=e^{-t} \sin x \quad, v(X, t)=e^{-t} \sin x
\end{aligned}
$$

\section{2) Coupled Burgers equation with space-fractional derivative}

\section{Example:}

$$
\left\{\begin{array}{l}
u_{t}=D_{x}^{2 \alpha} u+2 u D_{x}^{\alpha} u-D_{x}^{\alpha}(u v) \\
v_{t}=D_{x}^{2 \alpha} v+2 v D_{x}^{\alpha} v-D_{x}^{\alpha}(u v)
\end{array} \quad, 0<\alpha \leq 1\right.
$$

With the initial condition

$$
\left\{\begin{array}{l}
u(t, 0)=0 \quad, \quad v(t, 0)=0 \\
u_{x}(t, 0)=\frac{e^{-t}}{\Gamma(1+\alpha)}, v_{x}(t, 0)=\frac{e^{-t}}{\Gamma(1+\alpha)}
\end{array}\right.
$$

Using projected differential transform method of equation (11) we have 


$$
\begin{aligned}
& u_{t}(t, k)=\frac{\Gamma\left(1+2 \alpha+\frac{k}{q}\right)}{\Gamma\left(1+\frac{k}{q}\right)} u(t, k+2 \alpha q)+2 \sum_{m=0}^{k} \frac{\Gamma\left(1+\alpha+\frac{k}{q}\right)}{\Gamma\left(1+\frac{k}{q}\right)} u(t, m) u(x, k+\alpha q-m) \\
& -\sum_{m=0}^{k} \frac{\Gamma\left(1+\alpha+\frac{k}{q}\right)}{\Gamma\left(1+\frac{k}{q}\right)} u(t, m) v(x, k+\alpha q-m)-\sum_{m=0}^{k} \frac{\Gamma\left(1+\alpha+\frac{k}{q}\right)}{\Gamma\left(1+\frac{k}{q}\right)} v(t, m) u(x, k+\alpha q-m) \\
& v_{t}(t, k)=\frac{\Gamma\left(1+2 \alpha+\frac{k}{q}\right)}{\Gamma\left(1+\frac{k}{q}\right)} v(t, k+2 \alpha q)+2 \sum_{m=0}^{k} \frac{\Gamma\left(1+\alpha+\frac{k}{q}\right)}{\Gamma\left(1+\frac{k}{q}\right)} v(t, m) v(x, k+\alpha q-m) \\
& -\sum_{m=0}^{k} \frac{\Gamma\left(1+\alpha+\frac{k}{q}\right)}{\Gamma\left(1+\frac{k}{q}\right)} u(t, m) v(x, k+\alpha q-m)-\sum_{m=0}^{k} \frac{\Gamma\left(1+\alpha+\frac{k}{q}\right)}{\Gamma\left(1+\frac{k}{q}\right)} v(t, m) u(x, k+\alpha q-m) \\
& k=0, \alpha q, 2 \alpha q, \ldots . \\
& u(t, 2 \alpha q)=\frac{1}{\Gamma(1+2 \alpha)}\left[u_{t}(t, 0)-2 \Gamma(1+\alpha) u(t, 0) u(t, \alpha q)+\Gamma(1+\alpha) u(t, 0) v(t, \alpha q)\right. \\
& +\Gamma(1+\alpha) v(t, 0) u(t, \alpha q)]=0 \\
& v(t, 2 \alpha q)=\frac{1}{\Gamma(1+2 \alpha)}\left[v_{t}(t, 0)-2 \Gamma(1+\alpha) v(t, 0) v(t, \alpha q)+\Gamma(1+\alpha) u(t, 0) v(t, \alpha q)\right. \\
& +\Gamma(1+\alpha) v(t, 0) u(t, \alpha q)]=0 \\
& u(t, 3 \alpha q)=\frac{\Gamma(1+\alpha)}{\Gamma(1+3 \alpha)}\left[u_{t}(t, \alpha q)-2 \frac{\Gamma(1+2 \alpha)}{\Gamma(1+\alpha)} u(t, 0) u(t, 2 \alpha q)-2 \frac{\Gamma(1+2 \alpha)}{\Gamma(1+\alpha)} u(t, \alpha q) u(t, \alpha q)\right. \\
& +\frac{\Gamma(1+2 \alpha)}{\Gamma(1+\alpha)} u(t, 0) v(t, 2 \alpha q)+\frac{\Gamma(1+2 \alpha)}{\Gamma(1+\alpha)} u(t, \alpha q) v(t, \alpha q) \\
& \left.+\frac{\Gamma(1+2 \alpha)}{\Gamma(1+\alpha)} v(t, 0) u(t, 2 \alpha q)+\frac{\Gamma(1+2 \alpha)}{\Gamma(1+\alpha)} v(t, \alpha q) u(t, \alpha q)\right]=\frac{-e^{-t}}{\Gamma(1+3 \alpha)} \\
& v(t, 3 \alpha q)=\frac{\Gamma(1+\alpha)}{\Gamma(1+3 \alpha)}\left[v_{t}(t, \alpha q)-2 \frac{\Gamma(1+2 \alpha)}{\Gamma(1+\alpha)} v(t, 0) v(t, 2 \alpha q)-2 \frac{\Gamma(1+2 \alpha)}{\Gamma(1+\alpha)} v(t, \alpha q) v(t, \alpha q)\right. \\
& +\frac{\Gamma(1+2 \alpha)}{\Gamma(1+\alpha)} u(t, 0) v(t, 2 \alpha q)+\frac{\Gamma(1+2 \alpha)}{\Gamma(1+\alpha)} u(t, \alpha q) v(t, \alpha q) \\
& \left.+\frac{\Gamma(1+2 \alpha)}{\Gamma(1+\alpha)} v(t, 0) u(t, 2 \alpha q)+\frac{\Gamma(1+2 \alpha)}{\Gamma(1+\alpha)} v(t, \alpha q) u(t, \alpha q)\right]=\frac{-e^{-t}}{\Gamma(1+3 \alpha)} \\
& u(t, 4 \alpha q)=\frac{\Gamma(1+2 \alpha)}{\Gamma(1+4 \alpha)}\left[u_{t}(t, 2 \alpha q)-2 \frac{\Gamma(1+3 \alpha)}{\Gamma(1+2 \alpha)} u(t, 0) u(t, 3 \alpha q)-2 \frac{\Gamma(1+3 \alpha)}{\Gamma(1+2 \alpha)} u(t, \alpha q) u(t, 2 \alpha q)\right. \\
& -2 \frac{\Gamma(1+3 \alpha)}{\Gamma(1+2 \alpha)} u(t, 2 \alpha q) u(t, \alpha q)+\frac{\Gamma(1+3 \alpha)}{\Gamma(1+2 \alpha)} u(t, 0) v(t, 3 \alpha q)+\frac{\Gamma(1+3 \alpha)}{\Gamma(1+2 \alpha)} u(t, \alpha q) v(t, 2 \alpha q) \\
& +\frac{\Gamma(1+3 \alpha)}{\Gamma(1+2 \alpha)} u(t, 2 \alpha q) v(t, \alpha q)+\frac{\Gamma(1+3 \alpha)}{\Gamma(1+2 \alpha)} v(t, 0) u(t, 3 \alpha q) \\
& \left.+\frac{\Gamma(1+3 \alpha)}{\Gamma(1+2 \alpha)} v(t, \alpha q) u(t, 2 \alpha q)+\frac{\Gamma(1+3 \alpha)}{\Gamma(1+2 \alpha)} v(t, 2 \alpha q) u(t, \alpha q)\right]=0
\end{aligned}
$$




$$
\begin{aligned}
& v(t, 4 \alpha q)=\frac{\Gamma(1+2 \alpha)}{\Gamma(1+4 \alpha)}\left[v_{t}(t, 2 \alpha q)-2 \frac{\Gamma(1+3 \alpha)}{\Gamma(1+2 \alpha)} v(t, 0) v(t, 3 \alpha q)-2 \frac{\Gamma(1+3 \alpha)}{\Gamma(1+2 \alpha)} v(t, \alpha q) v(t, 2 \alpha q)\right. \\
& -2 \frac{\Gamma(1+3 \alpha)}{\Gamma(1+2 \alpha)} v(t, 2 \alpha q) v(t, \alpha q)+\frac{\Gamma(1+3 \alpha)}{\Gamma(1+2 \alpha)} u(t, 0) v(t, 3 \alpha q)+\frac{\Gamma(1+3 \alpha)}{\Gamma(1+2 \alpha)} u(t, \alpha q) v(t, 2 \alpha q) \\
& +\frac{\Gamma(1+3 \alpha)}{\Gamma(1+2 \alpha)} u(t, 2 \alpha q) v(t, \alpha q)+\frac{\Gamma(1+3 \alpha)}{\Gamma(1+2 \alpha)} v(t, 0) u(t, 3 \alpha q) \\
& \left.+\frac{\Gamma(1+3 \alpha)}{\Gamma(1+2 \alpha)} v(t, \alpha q) u(t, 2 \alpha q)+\frac{\Gamma(1+3 \alpha)}{\Gamma(1+2 \alpha)} v(t, 2 \alpha q) u(t, \alpha q)\right]=0
\end{aligned}
$$

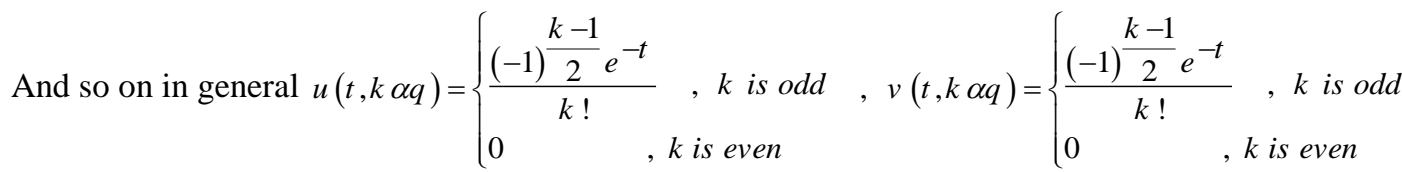

Substituting $u(X, k \alpha q)$ and $v(X, k \alpha q)$ into equation (5) we have

$$
\begin{aligned}
& u(t, x)=\sum_{k=1,3,5, \ldots}^{\infty} \frac{(-1)^{\frac{k-1}{2} e^{-t}}}{\Gamma(1+k \alpha)} x \alpha=\left(e^{-t}\right) \sum_{k=0}^{\infty} \frac{(-1)^{k}(x \alpha)^{2 k+1}}{(k \alpha+1) !} \\
& v(t, x)=\sum_{k=1,3,5, \ldots}^{\infty} \frac{(-1)^{\frac{k-1}{2} e^{-t}}}{\Gamma(1+k \alpha)} x k=\left(e^{-t}\right) \sum_{k=0}^{\infty} \frac{(-1)^{k}\left(x^{\alpha}\right)^{2 k+1}}{(k \alpha+1) !} \quad, \quad \alpha \rightarrow 1 \text { we get } \\
& u(t, x)=\left(e^{-t} \sum_{k=0}^{\infty} \frac{(-1)^{k}(x)^{2 k+1}}{(k+1) !}=e^{-t} \sin x, v(t, x)=\left(e^{-t}\right) \sum_{k=0}^{\infty} \frac{(-1)^{k}(x)^{2 k+1}}{(k+1) !}=e^{-t} \sin x\right.
\end{aligned}
$$

\section{Conclusion}

In this paper the projected differential transform method (P DTM) was used for coupled Burgers equation with time and space fractional derivative. The method is used in a direct way without using any linearization, perturbation, polynomials or restrictive assumptions in contrast to the current methods. The solution of our model equations are calculated in the form of convergent series with easily computable components. In summary, using projected differential transformation method to solve FPDE consists of three main steps. First, transformation FPDE in to algebra equation, second, solves the equations, finally inverting the solution of algebraic equations to obtain a closed form series solution.

\section{References}

[1] Adel Al.rabtah, redat suat Erturk, Shaher Momani: solution of fractional oscillator By using differential transform method. Comp. math. Appli 59 (2010) 1356-1362.

[2] A.M.A. El-Sayed, S.Z. Rida, A.A.M. Arafa, Exact solutions of fractional-order biological population model, Commun. Theor. Phys., 52, pp. 992-996, 2009 http://dx.doi.org/10.1088/0253-6102/52/6/04.

[3] Aydin Secer,Mehmet Ali Akinlar,Adem Cevikel, Efficient solution of system of fractional PDEs by the differential transform method, Advances in differential equations.

[4] Aytac Arikoglu, Ibrahim Ozkol: Solution of fractional differential equations by using differential transform method. Chaos. Solutions . Fractals 34(2007)1473-1481 http://dx.doi.org/10.1016/j.chaos.2006.09.004.

[5] Aytac Arikoglu, Ibrahim Ozkol: Solution of fractional integro - differential equations by using fractional differential transform method. Chaos. Solution. Fractals 40(2009) 251-529.

[6] F. Shakeri, M. Dehghan, Numerical solution of a biological population model using He's variational iteration method, Comput. Math. Appl., 54, pp. 1197-1209, 2007 http://dx.doi.org/10.1016/j.camwa.2006.12.076.

[7] Hüseyin Koçaka, Ahmet Yıldırımb;c, An efficient new iterative method for finding exact solutions of nonlinear time-fractional partial differential equations, Nonlinear Analysis: Modelling and Control, 2011, Vol. 16, No. 4, 403-414.

[8] P. Roul, Numerical solutions of time fractional degenerate parabolic equations by variational iteration method with Jumarie-modified Riemann-Liouville derivative, Math. Methods Appl. Sci., 34(9), pp. 1025-1035, 2011 http://dx.doi.org/10.1002/mma.1418.

[9] Salih M. Elzaki: Exact Solution of the (3+1) dimensional nonlinear Schrodinger equation using differential transform method, International Journal of Applied Mathematical Sciences ISSN 0973-0176 Volume 7, Number 1 (2014), pp. 41-46.

[10] Salih M. Elzaki: Solution of Burger's Equation Using Projected Differential Transform Method, International Journal of Mathematical Sciences, ISSN: 2051-5995, Vol.34, Issue.11498.

[11] Salih M. Elzaki: Exact Solution of Biological Population Model Using Projected Differential Transform Method, International Journal of Mathematical and Computer Modelling, ISSN: 2051- 4271, Vol.19, Issue.11124.

[12] Salih M. Elzaki: Solution of General System of Partial Differential Equation Using Projected Differential Transform Method, International Journal of Applied Mathematics, ISSN: 2051-5227, Vol.29, Issue.1 1271.

[13] Vedat Suat Ertrk. Shaher Momani: Solving systems of fractional differential equations using differential transform method. Journal of Compu. Appli. Math 215 (2008) 142-151. http://dx.doi.org/10.1016/j.cam.2007.03.029. 